\title{
Reconstruction of Transcription Regulatory Networks by Stability-Based Network Component Analysis
}

\author{
Xi Chen ${ }^{1}$, Chen Wang ${ }^{1}$, Ayesha N. Shajahan ${ }^{2}$, Rebecca B. Riggins ${ }^{2}$, Robert Clarke ${ }^{2}$, and \\ Jianhua Xuan ${ }^{1}$ \\ ${ }^{1}$ Department of Electrical \& Computer Engineering, Virginia Polytechnic Institute and State \\ University, Arlington, VA 22203 \\ ${ }^{2}$ Lombardi Comprehensive Cancer Center and Department of Oncology, Georgetown University, \\ Washington, DC 20057
}

\begin{abstract}
Reliable inference of transcription regulatory networks is still a challenging task in the field of computational biology. Network component analysis (NCA) has become a powerful scheme to uncover the networks behind complex biological processes, especially when gene expression data is integrated with binding motif information. However, the performance of NCA is impaired by the high rate of false connections in binding motif information and the high level of noise in gene expression data. Moreover, in real applications such as cancer research, the performance of NCA in simultaneously analyzing multiple candidate transcription factors (TFs) is further limited by the small sample number of gene expression data. In this paper, we propose a novel scheme, stabilitybased NCA, to overcome the above-mentioned problems by addressing the inconsistency between gene expression data and motif binding information (i.e., prior network knowledge). This method introduces small perturbations on prior network knowledge and utilizes the variation of estimated TF activities to reflect the stability of TF activities. Such a scheme is less limited by the sample size and especially capable to identify condition-specific TFs and their target genes. Experiment results on both simulation data and real breast cancer data demonstrate the efficiency and robustness of the proposed method.
\end{abstract}

\section{Keywords}

transcription regulatory network; network component analysis; stability analysis; transcription factor activity; target genes identification

\section{Introduction}

Transcription factors (TFs) are special proteins that control and affect the rate of downstream genes' mRNA expression. TFs can either activate or inhibit gene expression, usually by binding to short and highly conserved DNA sequences in promoter (or upstream) regions. Such transcriptional regulatory relationships can be explicitly described as transcriptional regulatory networks [1], consisting of TFs as regulators and downstream genes as targets.

(C) Springer-Verlag Berlin Heidelberg 2012

\{xichen86@vt.edu,topsoil@vt.edu,xuan@vt.eduans33@georgetown.edu,rbr7@georgetown.edu,clarke@ georgetown.edu\}. 
It has been known that a TF may play different regulatory roles to its downstream target genes or even has different downstream targets under different conditions [2]. Motivated by this understanding, many computational algorithms $[3,4]$ are proposed to discover condition-specific regulatory networks. By incorporating protein-DNA interaction (PDI) information into a linear latent variable model, several methods have also been proposed to solve the inference problem using regression or constraint regression like Network Component Analysis (NCA) [1] and Least Square (LS) regression [5]. Among them, NCA is a prominent method, which has mathematically derived identifiable conditions [6] and resulted in successful applications for several real biological problems [7, 8].

However, most of the existing methods including NCA do not consider the false positives in given network connections. Integrating gene expression data with inconsistent biological knowledge is a true challenge for the identification of TFs and their target genes. Another issue has to be considered is that NCA requires that the number of samples must be larger than the number of TFs [1]. However, in real practice, the number of gene expression data samples is usually smaller than the number of candidate TFs. Mainly because we don't know whether a certain TF is active or not in the condition where the gene expression data is sampled. Many candidate TFs should be tested in order to find the 'true' regulators. This limits the application of NCA to real biological studies, such as cancer research, for active TF identification.

In this paper, we propose a novel stability-based NCA (sNCA) approach to tackle the problem of regulatory network inference. By assuming that the consistency relationship between expression data and biological knowledge remains stable after small perturbations introduced to the prior knowledge of network topology, we can assign the knowledgederived estimation (i.e., transcription factor activity (TFA), regulatory strength (RS), etc.) an instability score (ISS), which represents the average distance of multiple estimations upon different perturbations. To solve the NCA limitation about the small sample size (relative to the number of TFs) in sNCA, at each time, we randomly sample a small number of TFs (<number of samples), calculate and store their ISS value. Then, after multiple times of randomly sampling, the averaged ISS value of each TF is calculated and used to prioritize all the TFs under investigation. Another scheme adopts local rank method. Here, we just change a small part of the selected TFs and only record the relatively stable TFs. After multiple times, the TFs counted for the most times are the global stable ones. Having the estimated activities of the TFs prioritized by sNCA, we further propose to use multivariate regression to rank the condition-specific target genes of active TFs.

To assess the performance of sNCA for TF identification, we have first applied it to simulation data in comparison with NCA and LS regression methods. The comparison results clearly demonstrate that sNCA outperforms the other two methods, especially when the network topology is incomplete or has false connections. Then, we apply sNCA to one breast cancer microarray dataset [9] to identify condition-specific TFs, aiming to provide insight into the underlying regulatory mechanism related to the development and progression of breast cancer. The experimental results show that our method can identify biologically meaningful TFs and target genes associating with estrogen signaling and action in breast cancer.

\section{Motif-directed NCA and Stability Analysis}

The sNCA approach is aimed to prioritize significant condition-specific TFs and their target genes, and uncover regulatory networks utilizing both gene expression data and binding motif information. Fig. 1 shows a flowchart of the proposed sNCA approach consisting of the following major components: (1) motif-direct NCA (m-NCA) and (2) stability analysis (SA). In the following subsections, we will give a description of each component in details. 


\subsection{Motif-directed NCA}

m-NCA scheme was proposed by us in [10] to infer regulatory networks, which utilizes sequence motif information to construct initial network connections, and later integrates with gene expression data to estimate the TFA and their downstream targets with the NCA algorithm. To construct the initial network, Match ${ }^{\mathrm{TM}}[11]$ can be used to search the transcription factor binding sites (TFBS) for their position-weighted matrices (PWMs) that can be extracted from the TRANSFAC 11.1 professional Database [12]. According to the PWMs, a motif score can be calculated for each TF-gene pair. These motif scores provide the initial connection information for m-NCA. Based on motif scores, we encode regulation relationship from $L$ TFs to $N$ target genes as a network connectivity pattern $B \in(0,1)^{N \times L}$, which is a binary matrix with element $b_{n, l}=1$ indicating potential regulatory relationship from $l$-th TF to $n$-th gene.

The gene expression data could be modeled as follows:

$$
x_{n}=\sum_{l=1}^{L} a_{n l} s_{l}+\gamma_{n}
$$

where $x_{n}=\left[x_{n 1}, \cdots, x_{n M}\right]$ and $\gamma_{n}=\left[\gamma_{n 1}, \cdots, \gamma_{n M}\right]$ are gene expression profile and noise vector of $n$-th gene, respectively. $M$ is the number of gene expression samples. $a_{n}=\left[a_{n 1}, \cdots\right.$ $\left.\cdot, a_{n L}\right]$ represents the regulatory strength for each TF on the $n$-th gene and $s_{l}=\left[s_{l 1}, \cdots, s_{l M}\right]$ is the hidden activity vector of $l$-th TF.

We further define regulatory strength (RS) matrix $A=\left[a_{1}, \cdots, a_{N}\right]^{T}$, and TFA matrix $S=$ $\left[s_{1}, \cdots, s_{L}\right]^{T}$ light of their biological implication. Finally, the gene expression data $X=\left[x_{1}\right.$, . $\left.\cdots, x_{N}\right]^{T}$ can be represented in a matrix-vector form as follows:

$$
X=A S+\Gamma \quad
$$

where $\Gamma=\left[\gamma_{1}, \cdots, \gamma_{N}\right]$ is the noise matrix, subject to zero-mean Gaussian distribution.

To solve Eq. (2), based on available biological knowledge $B$ and gene expression data, the NCA algorithm is designed to estimate $A$ and $S$ by minimizing the following fitting errors:

$$
(\widehat{A}, \widehat{S})=\underset{(A, S)}{\arg \min }\|X-A S\|_{2}^{2}, \quad \text { s.t. } \quad A \in \mathbb{Z}_{0}
$$

where $\mathbb{Z}_{0}$ is a regulatory matrix set, deriving from $B \in(0,1)^{N \times L}$ :

$$
\mathbb{Z}_{0} \triangleq\left\{A \in \mathbb{R}^{N \times L} \mid a_{n l}=0 \quad \text { for } \quad b_{n l}=0\right\}
$$

\subsection{Stability Analysis}

Stability analysis (SA) is used to identify true positive connection in the TF-gene networks. Actually, as TF binding motif is a relatively short sequence pattern, the topology obtained from motif information is quite noisy and contains many false positive connections. Since the initial topology information is often unreliable for specific TF-gene pairs, we are going to address the inconsistency between biological knowledge derived from DNA motif information and expression data through our proposed sNCA scheme. By adding small perturbations to the given topological connections, sNCA can be used to prioritize condition-specific TFs and their target genes. 
We aim to study the regulatory role of the $l$-th TF through its TFA estimation $s_{l}$, but the quality of biological knowledge, network information, is our concern. We can keep the expression unchanged and generated a perturbed $\mathbb{Z}_{0}^{\prime}$ by intentionally altering a small amount of connections in given $\mathbb{Z}_{0}$. We would expect a deviation (denoted as $d$ ) between estimated $s_{l}^{\prime}$ (based on the perturbed $\mathbb{Z}_{0}^{\prime}$ ) and original estimation $s_{l}$ (based on $\mathbb{Z}_{0}$ ). The rationale behind this is that the TFA estimation of a condition-specific TF should be more robust to a small amount of perturbation than any non-specific TF that generally lacks of support in data-network consistency.

Multiple perturbations are added to network topology for SA. With each individual perturbation, a different estimated TFA can be generated. $P$ independent perturbations on original $\mathbb{Z}_{0}$ obtain $P$ versions of $\mathbb{Z}_{p}, 1 \leq p \leq P$ and the degree of perturbation should be very small ( $\mathrm{FP}=\mathrm{FN}=\alpha \ll 1)$. Mathematically, we denote the perturbation function as follows:

$$
\mathbb{Z}_{p}=\text { Perturb }_{\alpha}\left(\mathbb{Z}_{0}, p\right), 1 \leq p \leq P
$$

The estimated TFA $\hat{s}_{l, p}$ of the $l$-th TF and associated RS $\hat{a}_{l, p}$ (with respect to expression data $X$ ) for the $p$-th perturbed regulatory matrix $\mathbb{Z}_{p}$ can be decomposed by NCA algorithm as

$$
\left(\widehat{a}_{l, p}, \widehat{s}_{l, p}\right)=N C A_{l-t h T F A}\left(X, \mathbb{Z}_{p}\right)
$$

where $N C A_{l-t h T F A}$ represents the $N C A$ estimation process for the $l$-th TF.

After $P$ times of above procedure, a stability measure, namely instability score (ISS) of TFA, is introduced here as follows:

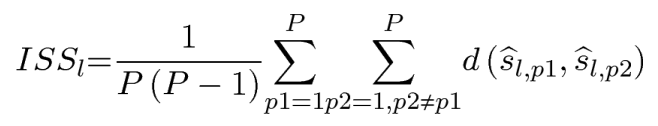

where the distance function $d\left(v_{1}, v_{2}\right)$ is defined as

$$
\begin{gathered}
d\left(v_{1}, v_{2}\right)=\log \left[d_{0}\left(v_{1}, v_{2}\right) /\left(1-d_{0}\left(v_{1}, v_{2}\right)\right)\right] \\
d_{0}\left(v_{1}, v_{2}\right)=\min \left(0.9, \cos \left(v_{1}, v_{2}\right), \cos \left(v_{1},-v_{2}\right)\right)
\end{gathered}
$$

where, $\cos (\cdot, \cdot)$ is the cosine distance. To prevent $d$ from approaching infinity when $d_{0}$ is close to 1 , here, we limit the maximum value of $d_{0}$ to 0.9 .

If the candidate TF number is larger than the sample number, to address an NCA limitation, here we randomly sample a small number of TFs (< sample number) at each time, then calculate and store their ISS values. After multiple times of randomly sampling, the averaged ISS value of each TF is calculated, which is further used to prioritize all the TFs. We call this process as 'average ISS based method'.

Considering another selection scheme, first, we randomly sample a small number $L$ of TFs. Then another $L^{\prime}$ TFs are selected from the rest ones, and totally $L+L^{\prime}$ TFs ( $<$ sample number) are tested as Eq. (6). Based on their ISS value, the top $L$ TFs are retained and the count for each is added by 1 . In each round, we randomly select a new set of $L^{\prime}$ TFs and do stability analysis. After multiple times of sampling, the count of each TF is used to prioritize all the TFs. This is named as 'rank based' sampling method, compared to the 'average ISS based' sampling scheme. 
Based on identified TFs, we further utilize multivariable regression scheme to identify target genes controlled by them. For the $n$-th gene, we use the activities of its potential regulators, TFA, to regress its expression profile $x_{n}$ and calculate the resulted $p$-value of regression coefficient. $p$-value reflects how well the gene expression data can be fitted by corresponding TFAs. Here, principle component analysis (PCA) [13] is adopted to estimate the main TFA $\hat{s}_{n, l}$ among $P$ times of m-NCA results.

With the estimated TFA matrix $\hat{S}$, considering formula (2), by using the least square errors regression method, the associated RS matrix $\hat{A}$ is obtained as:

$$
\widehat{A}^{T}=\left(\widehat{S} \widehat{S}^{T}\right)^{-1} \widehat{S} X^{T}
$$

We simply definite the relevance score of downstream gene (RSDG) based on its average significance level according to TFA regression analysis.

$$
R S D G_{n}=\frac{-\sum_{l=1}^{L} \log \left(p-\text { value }\left(\widehat{a}_{n, l}\right)\right)}{\operatorname{count}\left(\widehat{a}_{n, l} \neq 0\right)}
$$

where function $\operatorname{count}(\cdot)$ is to count the number of regulatory strength $\hat{a}_{n, l}$ which does not equal to zero. The higher the RSDG is, the more likely this gene is truly regulated by $l$-th TF under this condition.

To summarize, for inference of transcription regulatory networks using the proposed stability-based approach, we propose two consecutive steps: 1) identify condition-specific TFs by sNCA; 2) identify condition-specific downstream target genes based on stable TFA regression.

\section{Simulation and Experiment Results}

\subsection{Simulation Data}

To test the performance of the sNCA approach, we generate 100 different simulated regulatory networks consisting of $30 \mathrm{TFs}$ and 500 target genes. On average, each gene is regulated by 3 TFs. The gene expression data is formed by using the model defined in formula (1), where the RS and TFA both follow Gaussian distribution. The purpose of this study is to first identify true regulators and then their significant target genes.

Here we design two kinds of simulation data. For 'none-overlap' case, 50 gene expression data samples are generated as regulated by $15 \mathrm{TFs}$, and another $15 \mathrm{TFs}$ have no impact on the gene expression data. So just some unrelated network information is added to the 'true' network, which has 'none overlap' with the regulatory mechanism of the gene expression data. Comparably, for the 'overlap' case, the gene expression samples are regulated by all 30 TFs, within which $15 \mathrm{TFs}$ have false positive/false negative connections of 5\% and the rest is contaminated with false connections of $30 \%$. Here, apparently, the network with high false connections has 'overlap' with the regulatory mechanism of the gene expression data. Actually, sometimes we don't know whether a certain TF has any relationship with condition specific gene expression data. At this time, in order to not miss important TFs, we have to enlarge the range of candidate TFs. If the added TFs have no relationship with gene expression data, like the 'none-overlap' case, the true regulatory mechanism is not affected. But if the added TFs have certain relationship with the data but with high noise in their network topology, the false positive of the network information is increased, as the 'overlap' case. Here, we compare sNCA TF identification performance with NCA and LS regression under different SNR conditions. The performance is comprehensively measured by receiver 
operating characteristic studies; in particular, the area-under-ROC curve (AUC) is used, as shown in Fig. 2 (a) and (b).

In Fig.2, we can see that the LS regression always has the worst performance compared to the others. It is reasonable because there is no refining estimation about the regulation strength between TFs and their target genes. In Fig.2 (a), 'none-overlap' case, NCA has a comparable performance as sNCA, mainly due to the correct network topology information assumed in the 'none-overlap' case. However, if the signal to noise ratio (SNR) is low, the consistency between gene expression data and network topology is affected severely by the noise in expression data, which makes NCA cannot estimate the TFA with high accuracy. So its performance comes down close to the LS regression. This proves that NCA algorithm is quite sensitive to inconsistency between the network topology and expression data. In Fig. 2 (b), the NCA performs much worse than sNCA for the 'overlap' case. Apparently, the false connections in network topology severely affect NCA algorithm accuracy. Comparing the performance of sNCA in Fig.2 (a) and (b), we can find that if the network is more complicated (regulated by 15 vs. 30 TFs), the sNCA scheme is more robust, although false connections exist. Therefore, sNCA improves the robustness of the TF identification compared to the NCA scheme, and could potentially be more effective when applied to the inference of complex networks.

Further, sNCA is used to identify the target genes of stable TFs, where neither NCA nor LS regression could work. The performance of target gene identification by sNCA in the above two simulation data sets is summarized in Table 1.

Considering the 5\% false connection in the 'none-overlap' case, the AUC value of target gene identification comes close to 0.95 when the SNR is high. In the 'overlap' case, the TF gene regulation mechanism is affected more by the high level false connections. But the AUC value for target gene identification does not degrade significantly. This point proves that sNCA could the estimate TFA with high robustness against the inconsistency between gene expression data and network topology. And also, the RSDG is a proper measure to prioritize the target gene.

To investigate the small sample number vs. large TF number problem, here, we increase the number of TFs to 80, and keep sample number still 50. For the 'none-overlap' case, gene expression data is regulated by 40 TFs, and the rest TFs have no impact on the gene expression data. For the 'overlap' case, gene expression data is regulated by 80 TFs, within which 40 TFs are contaminated with $30 \%$ of false connections. Because NCA scheme requires that the sample number should be larger than the TF number, so it cannot be used in this case due to its theoretical limitation. As mentioned in section 2.2, sNCA could solve this problem by sampling a subset of TFs and calculating the mean ISS value after multiple randomly sampling, or counting the relatively stable TFs within each round of sampling. Thus, we test the TF identification performance of sNCA with both sampling methods, which are shown in Table 2.

Compared to Fig. 2 and Table 1, in Table 2, for the 'none-overlap' case, although the performance of TFs identification by sNCA degrades slightly due to the incomplete network knowledge, it could still keep a relatively high accuracy to identify true regulatory modules. Here the 'average ISS based' method and rank based method (not listed here) have similar performance. For the 'overlap' case, if we use the average ISS value after multiple times of sampling to identify stable TFs, the AUC performance degrades more compared to the rank based method. So 'rank based' method could identify global optimal TFs from local prioritization selection. Although the overall performance degrades when the sample number is smaller than the number of TFs, to certain extent, the sNCA approach can overcome the 
limitation of NCA and provide us a possible solution to analyze multiple candidate TFs simultaneously with limited samples, which is especially useful in cancer research.

\subsection{Breast Cancer Expression Data}

The sNCA scheme is further applied to a breast cancer expression data set [9] to identify condition specific TFs and significant target genes. According to the survival time, we group the gene expression data samples into two groups, 49 samples in 'early' group ( $<3$ years) and another 51 samples in 'late' group ( $>8$ years). T-test is used to select top $\sim 4,000$ genes that have different patterns between the early and late groups. Then, these genes are further divided into two clusters. Genes in 'Cluster 1' have higher expression values in the early group, and comparatively, genes in 'Cluster 2' express higher in the late group. From literature and biological databases, we have nearly $240 \mathrm{TFs}$, which are likely associated with breast cancer and estrogen signaling. Finally, sNCA is applied to cluster 1 and 2 respectively, to test each candidate TF.

Actually, we are more focusing on the TFs whose activities have different pattern between the two sample groups. In this case, their target genes are more probably associated with the survival time of breast cancer patients. Here, defining a phenotype vector $C=\left[c_{1}, \cdots, c_{M}\right]$ for $M$ samples (100 in our case), with - 1 for each sample in the early group and 1 for each sample in the late group. Among all the TFs selected by sNCA, the coefficient (Coef.) of the $l$-th TF's activity $s_{l}$ and vector $C$ is calculated as

$$
\text { Coef } f_{l}=\sum_{m=1}^{M} s_{l, m} c_{m} / \sqrt{\operatorname{var}\left(s_{l}\right) \operatorname{var}(C)}
$$

The absolute value of Coef. is used to further refine our selected TFs pool. Based on the ISS value from sNCA and the Coef., the top stable TFs are listed in Table 3.

As shown in Fig. 3 (a), these tumor and breast cancer related TFs have direct relationships through protein-protein interaction (PPI) and protein-DNA interaction (PDI). Here, transcription factor ESR1 and SP1 (the green bin in Fig.3 (a)) are added in to make the network more complete. Although they are not covered in Table 3, their important neighbors are ranked quite high through our analysis. Also, some existing biology literatures provide strong support to our computational results. Among all the selected TFs, E2F1, a key transcription factor involved in proliferation, differentiation and apoptosis has been observed to associate with breast cancer favorable outcome [14]. CREB1 is a co-activator of ESR1. ELK1 is a subclass of ETS family and a breast cancer study has shown over expression of ETS1 is indicative of poor prognostics [15]. JUN is associated with drug resistance [16] and CEBPB is already well known to be associated with breast cancer [17]. WT1 also has relationship with breast carcinoma [18]. Although two important TFs PPARA and PBX1 are not connected directly to the other components in our network, these two TFs have intense relationship with AP1. AP1 is a well known TF that has been identified in breast cancer research [19]. POU2F1 is a member of the POU family of TFs and is involved in hormonal signals [20].

Moreover, based on Eq. (11), the candidate target genes are sorted by the RSDG value. In the Fig. 3 (b), we show the expression pattern of the significant target genes identified by sNCA for several TFs appearing in the network. These genes are regulated by TFs with significant different activities between the early and late groups. So they also show significant and consistent pattern associating with their regulators. To certain extent, these genes have some relationship with the patient survival time. To test the prediction performance of these genes, support vector machine (SVM) is adopted here. 5-fold cross 
validation is applied to the 100 breast cancer samples in the early and late groups. The receiver operator characteristics (ROC) curve and the Kaplan-Meier (KM) survival time plot are shown in Fig. 4 (a) and (b), respectively.

From the cross validation ROC curve in Fig. 4(a), the AUC value of the prediction performance of genes identified by sNCA is 0.8106 , compared to 0.72 of the genes randomly selected from the $\sim 4,000$ genes (from t-test). Also, when the sensitivity (true positive rate) is fixed at 0.70 , the specificity is 0.79 for sNCA identified genes and 0.66 for randomly selected genes. Considering Fig. 4(b), the KM plot, when the survival time is at 3 years, the late group survival portion is 0.72 , compared to 0.22 for the early group. The division of the two groups is quite well. So from computational point of view, the genes that the sNCA scheme identifies are highly associated with the survival time of breast cancer patients.

Further, we selected the top 5\% genes ( 200) ranked by their RSDG value to do functional analysis and annotation. Based on Ingenuity Pathway Analysis (IPA) system [21], the functional annotation of these genes can be found in Table 4.

Table 4 shows that a high portion of the target gene can be functionally grouped together. Through the annotation, we know that these processes have intense relationship with cancer progression. Also, considering that our data set is estrogen-dependent breast cancer, many transcription factors included in estrogen-receptor signaling pathways (e.g., CREB1, STAT5, ATF2, JUN, ELK1) appear in our identified TFs and target genes. Also, from bottom to up, CREB1, CEBPA, CEBPB, JUN, ETS1 and E2F1 are highlighted by our identified target genes in IPS system. This further proves that our identified TFs and target genes support each other well.

\section{Conclusions}

In this paper, we have proposed a novel approach, stability-based network component analysis (sNCA), to prioritize TFs and their target genes simultaneously. The sNCA approach overcomes a major limitation in many existing methods as caused by the inconsistency between gene expression data and TF-gene binding knowledge. With a proper stability analysis procedure, the sNCA approach also overcomes the limitation of NCA due to the small sample size of gene expression data. Through simulation studies, the proposed sNCA approach outperforms both NCA and LS regression, especially when the network topology information is incomplete or with a high level of noise. The experimental results from an analysis of real breast cancer data have further demonstrated that SNCA can help identify biologically meaningful regulatory networks associated with the development and progression of breast cancer.

\section{Acknowledgments}

This work was supported by the National Institutes of Health (CA139246, CA149653 CA149147 and NS29525-18A).

\section{References}

1. Liao JC, Boscolo R, et al. Network component analysis: reconstruction of regulatory signals in biological systems. Proc. Natl. Acad. Sci. USA. 2003; 100(26):15522-15527. [PubMed: 14673099]

2. Luscombe NM, Babu MM, et al. Genomic analysis of regulatory network dynamics reveals large topological changes. Nature. 2004; 431:308-312. [PubMed: 15372033]

3. Chen L, Xuan J, et al. Multilevel support vector regression analysis to identify condition specific regulatory networks. Bioinformatics. 2010; 26(11):1416-1422. [PubMed: 20375112] 
4. Rverter A, Hudson NJ, et al. Regulatory impact factors: unraveling the transcriptional regulation of complex traits from expression data. 2010; 26(7):896-904.

5. Nguyen DH, Haeseleer PD. Deciphering principles of transcription regulation in eukaryotic genomes. Mol. Syst. Biol. 2006:2.

6. Boscolo R, Sabatti C, et al. A generalized framework for network component analysis. IEEE/ACM Trans. Comput. Biol. Bioinformatics. 2005; 2(4):289-301.

7. Brynildsen MP, Liao JC. An integrated network approach identifies the isobutanol response network of Escherichia coli. Mol. Syst. Biol. 2009; 5:277. [PubMed: 19536200]

8. Ye C, Galbraith SJ, et al. Using network component analysis to dissect regulatory networks mediated by transcription factors in yeast. PLoS Comput. Biol. 2009; 5(3):e1000311. [PubMed: 19300475]

9. Loi S, Haibe-Kains B, et al. Definition of clinically distinct molecular subtypes in estrogen receptorpositive breast carcinomas through genomic grade. J. Clin. Oncol. 2007; 25(10):39-46.

10. Wang C, Xuan J, et al. Motif-directed network component analysis for regulatory network inference. BMC Bioinformatics. 2008; 9:S.1, S21.

11. Kel AE, Gossling E, et al. MATCH: A tool for searching transcription factor binding sites in DNA sequences. Nucleic Acids Res. 2003; 31(13):3576-3579. [PubMed: 12824369]

12. Matys V, Kel-Margoulis OV, et al. Transfac and its module TransCompel: transcriptional gene regulation in eukaryotes. Nucleic Acids Res. 2006:D108-D110. [PubMed: 16381825]

13. Jolliffe, IT., et al. Principle component analysis. 2nd edn.. Springer; NY: 2002. p. 167-198.

14. Vuaroqueaux V, et al. Low E2F1 transcript levels are a strong determinant of favorable breast cancer outcome. Breast Cancer Research. 2007; 9:R33. [PubMed: 17535433]

15. Buggy Y, Maguire TM, et al. Over expression of the Ets-1 transcription factor in human breast cancer. Br. J. Cancer. 2004; 91(7):1308-1315. [PubMed: 15365563]

16. Daschner PJ, Ciolino HP, et al. Increased AP-1 activity in drug resistant human breast cancer MCF-7 cells. Breast Cancer Res. Treat. 1999; 53(3):229-340. [PubMed: 10369069]

17. Shackleford TJ, et al. Stat 3 and CCAAT/enhancer binding protein beta (C/EBP-beta) regulate Jab1/CSN5 expression in mammary carcinoma cells. Breast Cancer Research. 2011; 13:R65. [PubMed: 21689417]

18. Domfeh AB, et al. WT1 immunoreactivity in breast carcinoma: selective expression in pure and mixed mucinous subtypes. Modern Pathology. 2008; 21:1217-1223. [PubMed: 18469795]

19. Shen Q, et al. The AP-1 transcription factor regulates breast cancer cell growth via cyclins and E2F factors. Oncogene. 2008; 27:366-377. [PubMed: 17637753]

20. Kakizawa T, et al. Silencing mediator for retinoid and thyroid hormone receptors interact with octamer transcription factor-1 and acts as a transcriptional repressor. J. Biol. Chem. 2001; 276:9720-9725. [PubMed: 11134019]

21. Ficenec D, et al. Computational knowledge integration in biopharmaceutical research. Brief. Bioinformatics. 2003; 4:260-278. [PubMed: 14582520] 


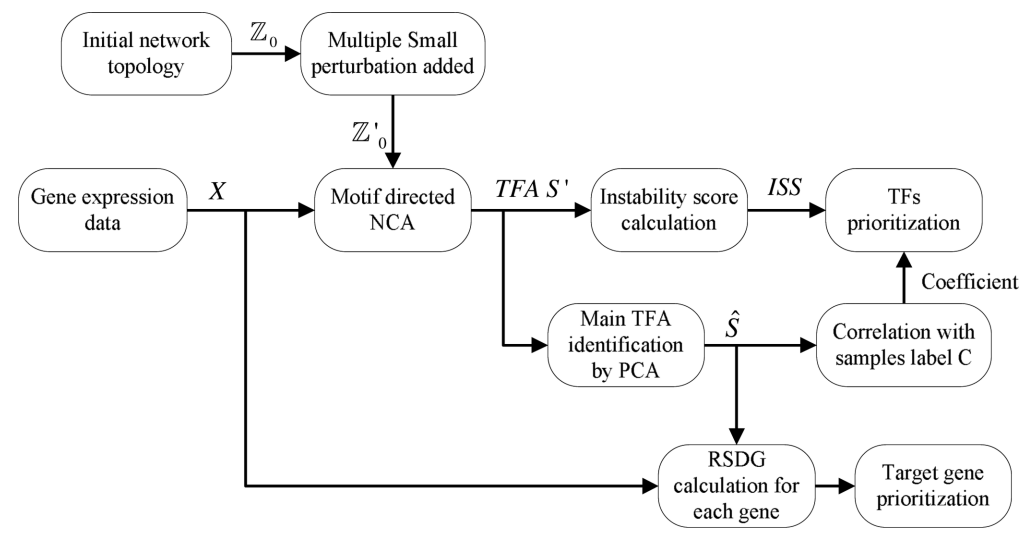

Fig. 1.

Flowchart of the proposed sNCA approach 

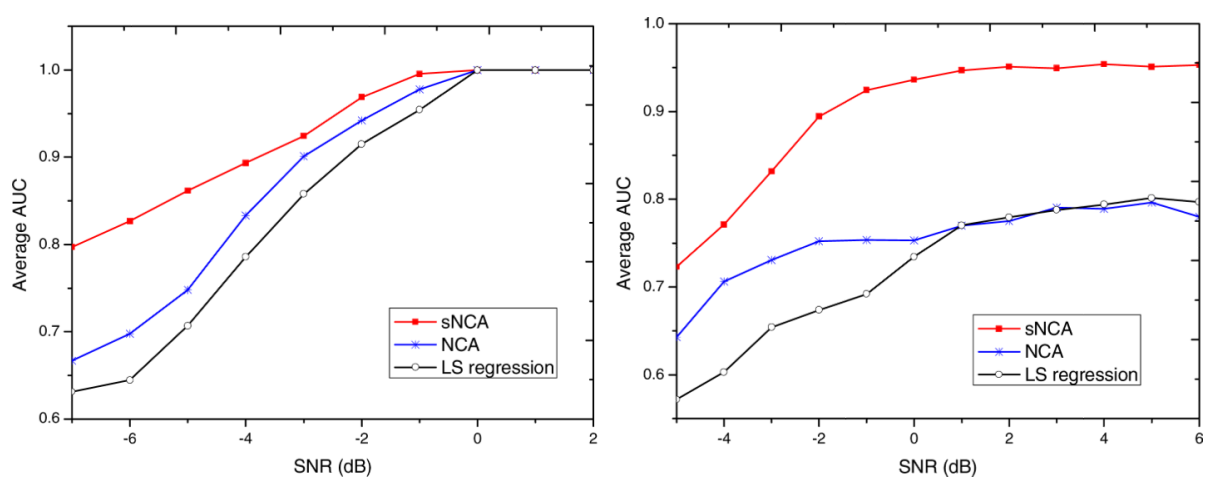

Fig. 2.

Comparison of TF identification performances by ROC studies: (a) AUC of the 'none overlap' case; (b) AUC of the 'overlap' case 

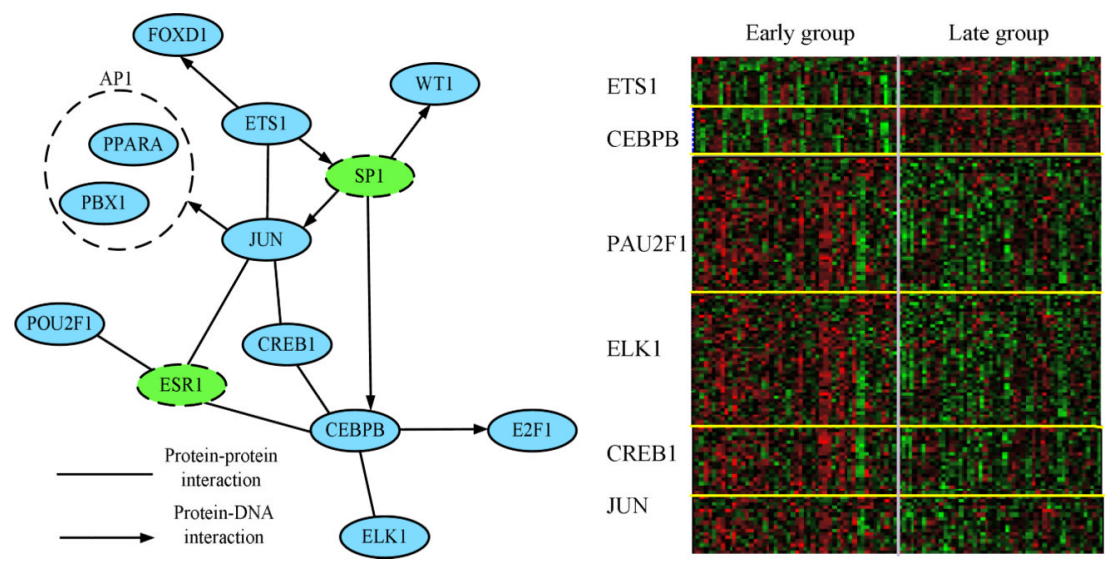

Fig. 3.

(a) Identified TFs network and (b) associated target genes pattern 

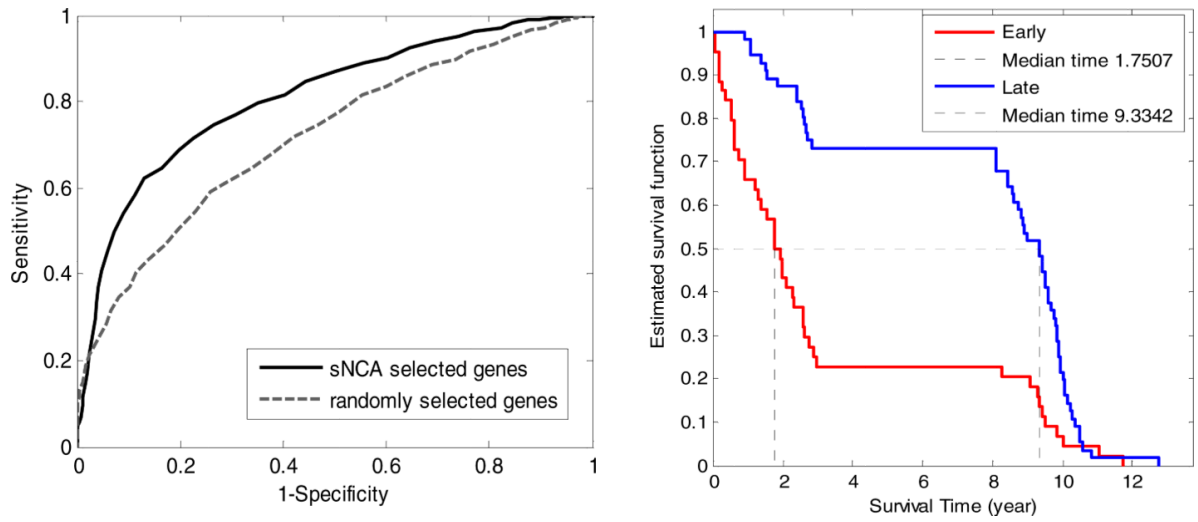

Fig. 4.

Prediction performances of 200 downstream target genes. (a) Comparison of ROC performance, (b) KM plot of the genes selected by SA. 


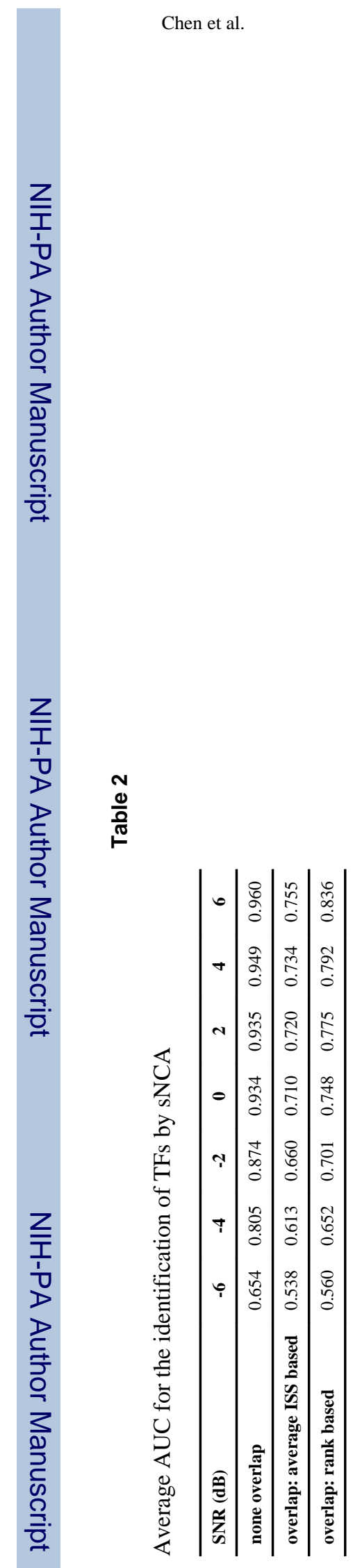

Page 15

IEEE/ACM Trans Comput Biol Bioinform. Author manuscript; available in PMC 2014 January 22. 
Table 4

Summary of functional groups of sNCA identified target genes

\begin{tabular}{lc}
\hline Functional annotation & Molecules \\
\hline Cancer & 81 \\
DNA replication, repair & 31 \\
Cell death & 46 \\
Cellular growth and proliferation & 39 \\
Cell cycle & 33 \\
\hline
\end{tabular}

